\title{
Application of Magnetic Nanoparticles in Pharmaceutical Sciences
}

\author{
Michał Piotr Marszałt
}

Received: 30 July 2010 / Accepted: 14 September 2010 / Published online: 22 September 2010

(C) The Author(s) 2010. This article is published with open access at Springerlink.com

KEY WORDS magnetic beads - magnetic bioseparation . magnetic nanoparticles

\section{INTRODUCTION}

Micro- and nano-sized magnetic support is a recently developed revolutionary technology for bioseparation, especially for ligand fishing, protein, enzyme, DNA, RNA and cell isolation or purification. Magnetic nanoparticles (MPs) are referred to by various synonyms, such as magnetic beads (MB) or micro- and nano-sized magnetic beads. They are also called ferrofluids or magnetic fluids, meaning colloidal suspensions of magnetic particles in a liquid carrier. Generally, these particles are part of nanotechnology, which can be defined as engineering of functional systems on a molecular scale. It has the potential to create many new materials and devices with a wide range of applications across the biomedical, chemical, electronic and mechanical fields.

Their superparamagnetic properties have opened promising new perspectives for their application in several areas. The pioneering "medical" application in the treatment of lymphatic nodes and metastases based on injecting "metallic particles" preheated in a magnetic field was first published by Gilchrist et al. in 1957 (1). Since then, magnetic particles have been modified by coating with antibodies, enzymes, proteins or specific ligands that enable

\footnotetext{
M. P. Marszałt $(\square)$

Department of Medicinal Chemistry, Faculty of Pharmacy

Collegium Medicum, Nicolaus Copernicus University

ul. M. Skłodowskiej-Curie 9

85-094 Bydgoszcz, Poland

e-mail: mmars@cm.umk.pl
}

them to bind to other biologically active compounds or receptors on the cell surface. In the last decade, magnetic particles have been increasingly used as a promising technique for a wide spectrum of biomedical applications. The number of publications presenting original studies on using magnetic beads is increasing year by year (Fig. 1). The growing interest in the magnetic carrier is focused on biochemistry, molecular biology and medical specialties. Because of the superparamagnetic properties and microand nano-dimensions, magnetic nanoparticles can be used to isolate any target and linked to various manual and automated applications (2-4). Innovative research has produced a new application for magnetic nanoparticles that carries an exciting biomedical and bioengineering potential, for instance, cell molecule and nucleic acid separation, immunoassays, pathogen detection, protein purification, gen mutation analysis and magnetic-forcebased tissue engineering $(5,6)$. Also, the interest in the potential application of the magnetic technique in pharmacy is significantly growing. It is currently being recognised that this magnetic nanotechnology could play an important role in this area.

\section{DRUG DISCOVERY}

The process of drug discovery in the modern scientific aspect is very complex. It integrates many disciplines, including biotechnology, medicine and pharmacology. For years, drug discovery has also focused on identification of unknown biomolecular interactions with known targets. A number of established methods are used in evaluating the binding of ligands/drugs to receptors and proteins, such as equilibrium dialysis, ultrafiltration, ultracentrifugation, bioaffinity chromatography and other spectroscopic methods. 


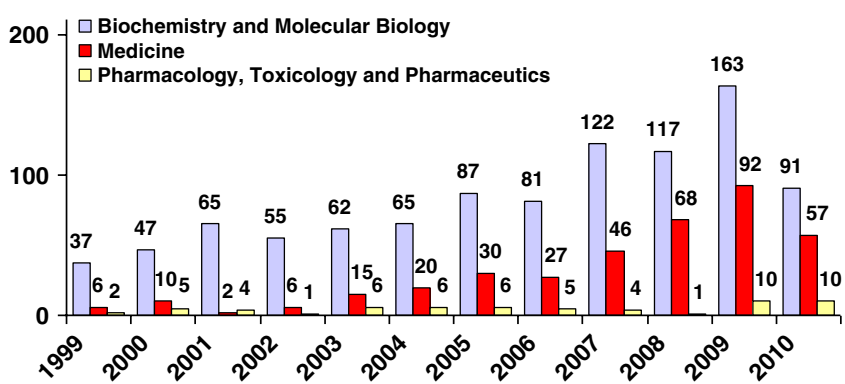

Fig. I The number of publications on the subject "Magnetic Beads" and "Biochemistry and Molecular Biology, Medicine or Pharmacology, Toxicology and Pharmaceutics" identified by Scopus (http://www.scopus.com/ home.url).

Also, the BIAcore system and surface plasmon resonance (SPR) are the two most popular recently technologies for real-time biomolecule interaction analysis. Because of the different modifications of multifunctional surface of the magnetic particles, these approaches are practically useful in this area.

The objective of the recent study was to adapt immobilized human serum albumin (HSA) onto the surface of magnetic beads for the purpose of "ligand fishing" (3). These beads correctly isolated a known binder from a mixture of known compounds, and the whole experiment was carried out manually using a magnet and automatically using the Magtration System. Furthermore, the magnetic separation technique was extended to the protein-ligand and protein-protein interaction using magnetic beads with immobilized heat-shock protein $90 \alpha(\mathrm{Hsp} 90 \alpha)$ (2). The advantage of this system is that the Hsp90 $\alpha$-coated magnetic beads can isolate interacting partners (ligand and proteins) from complex mixtures and cellular extracts (e.g. from KU-812 cells) in less than 15 min, unlike coimmunoprecipitation methods, which take several hours to recover the protein of interest. Presently, the extension of the magnetic beads method to multiple complex cellular extracts and complex plant extracts is being investigated. The most recent study demonstrates that ligand fishing based on biological functionalised $\mathrm{MB}$ is an effective and convenient way to identify and isolate bioactive small molecules from botanical extracts (Camptotheca acuminata and Dioscorea nipponica), and the process has a significant structure specifity $(7,8)$.

Using a similar concept, protein-coated magnetic beads were used as a tool for a rapid drug-protein binding study (4). Knowledge of serum protein binding behaviour as a significant component of blood is very important for the rational use of drugs. An extensive understanding of the ligand/drug-protein interaction may be of special importance for the modelling of pharmacokinetics, including possible drug-drug interaction. In this context, magnetic beads with immobilized HSA have been successfully used to determine the affinity of known drugs for the protein (4). Moreover, the preliminary competition experiments suggest that protein-coated magnetic beads can be employed in drug-drug interaction studies. However, the validation of the proposed method is being investigated.

\section{DRUG DELIVERY}

Advances in many fields are converging to make the commercialisation of advanced drug delivery concepts possible (6). Innovative drug delivery devices should protect labile active ingredients, precisely control drug release kinetics, and minimise the release of the drug to nontarget sites.

The major advantage of ferromagnetic materials $\left(\mathrm{Fe}_{3} \mathrm{O}_{4}\right)$ and maghemite $\left(\gamma-\mathrm{Fe}_{2} \mathrm{O}_{3}\right)$ over other materials with a high magnetic moment is the possibility for in vivo applications (6). Other materials, such as cobalt and nickel, can cause oxidative stress or long-term changes in enzyme kinetics. Hence, their use should be limited in biomedical applications.

The use of magnetic nanoparticles as drug carriers in targeted therapy provides huge opportunities in cancer treatment, as the use of such carriers considerably reduces the side effects of conventional chemotherapy. For drug targeting, they are able to cumulate in desired locations within the body with the help of a magnet. In 1978, magnetic particles were first applied as a new class of drug target. The tool was based on albumin microspheres with entrapped $\mathrm{Fe}_{3} \mathrm{O}_{4}$ and doxorubicin. A novel carrier system allowed for the accumulation of local chemotherapeutic agent which was comparable to that achieved by the administration of a 100-fold higher dose of the drug. Other results indicate that the new targeting system of specific drug delivery based on the "magnetic granules" might be useful for local chemotherapy of oesophageal cancer in rabbits administered via the oral route. Another interesting application has utilised iron oxide nanoparticles covered by starch polymers which makes the ferrofluids tolerable to healthy tissues and cells. The time-dependent distribution of the ferrofluids as a carrier for mitoxantrone within the body was already investigated by labelling the nanoparticles with radioactive isotope ${ }^{59} \mathrm{Fe}(9)$. Also, electron microscope investigations have shown that the ferrofluids do not only concentrate in the cancer tissue but also penetrate into the tumour cells.

The use of magnetic beads can significantly improve hyperthermia cancer treatment. This therapy involves raising the temperature of the target tissue to $43-46^{\circ} \mathrm{C}$, which increases its sensitivity to chemo- and radiotherapy and may additionally stimulate activities of the host immune system (10). Magnetic hyperthermia relies on the introduction of ferro- or superparamagnetic particles into 
the tumor tissue. Under an applied magnetic field, magnetic energy is converted to thermal energy, which destroys cancer tissues. Cancer-specific binding agents could include antibodies, hormones, and other exo- and endogenous substances. Attaching these agents, such as estrogen, testosterone, and thyroid hormones, to magnetic nanoparticles has promising implications for magnetic fluid hyperthermia treatment $(\mathrm{MFH})$ of breast, prostate, and thyroid cancer, respectively. Recently, monoclonal antibodies to cancer cell surface antigens and modified viruses that are able to bind with receptors on certain types of cancer cells are being considered as novel potentially binding agents coupled with magnetic particles. This tool could be successfully used to locate cancer cells and deliver chemotherapeutics directly to "cancer" location at elevated temperatures.

A recent study published in Nature Nanotechnology reports for the first time target aerosol delivery to the lung achieved with aerosol droplets comprising superparamagnetic iron oxide nanoparticles in combination with a target-directed magnetic gradient field (11). Standard therapy with cytotoxic drugs relies on the attainment of high drug concentrations at the affected site, which is particularly difficult in lung cancer (12). Thus, the novel and promising method could be used to accumulate effective drug doses in the affected lung areas and reduce undesirable effects. As the in vitro study has illustrated the enhanced deposition of high aspect ratio aerosols in the small airways using magnetic field alignment, in a recent study, computational fluid dynamics (CFD) simulations of magnetic aerosol transport and deposition were conducted and compared with the experimental findings (13). The demonstrated excellent predictive power of the CFD model suggests that inhalation delivery systems for in vivo selective respiratory drug delivery can potentially be designed based on observable magnetic and aerosol properties. The magnetic targeted carriers (MTCs) have also been proposed and developed to submicron-sized ferromagnetic particle preparation containing relaxant drugs for local anesthesia and improve targeting efficiency of pharmaceutical agents in the treatment of carcinoma in rabbits (14).

Furthermore, recent clinical research shows that exposure to iron nanoparticles increases endothelial cell permeability caused by oxidative stress resulting from reactive oxygen material (15). This phenomenon offers fascinating opportunities for magnetic beads in personalised cancer therapies.

\section{CELL AND GENE THERAPY}

Cell therapies are based on biological agents involving stem cells or immune cells to be administered to patients for the treatment of several diseases. The development of noninvasive imaging techniques to monitor cell migration to target tissues is of particular importance for cell-based therapies. Monitoring magnetically labeled cells with magnetic resonance imaging (MRI) has increased our understanding of cellular migration and the pathophysiology of diseases in experimental models and in vivo (16). Recently, the potential clinical application of anionic magnetic nanoparticles (AMNPs), ultrasmall paramagnetic iron oxides (USPIOs) and superparamagnetic iron oxide nanoparticles (SPIONs) has been demonstrated to visualise cell migration during MRI monitoring of cellular therapies $(17,18)$. These agents cause a strong source of contrast resulting from the increased differences in signal intensities that each tissue produces in response to the applied radio frequency pulses. Recent reports describe utilising small contrast agents to track the migration of stem cells, immune cells and other cells in numerous animal models of malignancy, angiogenesis, ischaemia, organ failure, autoimmune diseases and transplantation rejection (17). Tracking labelled cells using magnetic particles with MRI has clearly demonstrated its usefulness in evaluating promising cell-based therapies in preclinical models. However, their translation to the clinical practice is being considered in the near future (17).

The successful magnetic microsphere delivery approach has been used in gene therapy to reduce the incidence of adverse events, particularly in the case of viral and retroviral vectors. Gene therapy is a potential therapeutic tool that requires effective gene delivery. However, the most common methods for delivering genes into cell is associated with two potential dangers: oncogenesis and inflammation. This therapeutic strategy, based on magnetic technology, is an alternative method of viral gene delivery. Moreover, magnetic reporter genes inserted into cells result in expression of iron storage proteins that can be detected by MRI. The novel approach for improving viral DNA delivery system has been studied using modified cationic chitosan-coated iron oxide nanoparticles (Nac-6-IOPS) (19). The advantage of this system is the reduction of viral doses, time of infection and viral side effects. In another study, the enhancement of non-viral gene transfer was achieved by Lipofectamine $^{\text {TM }} 2000$ or cationic lipid 67 (GL67)/plasmid DNA (pDNA) liposome complexes coupled with superparamagnetic particles (20). A novel non-viral delivery system based on a polyethylenimine coated on the surface of bacterial magnetic nanoparticles (BMPs) has been developed with high transfection efficiency and low toxicity, which presents an attractive strategy for gene therapy and DNA vaccination (21).

The nanoparticle technology is highly novel and offers many possibilities for future development. Although the particles possess useful properties for biomedical applica- 
tions, they also carry potential health risks. Generally, the nanomaterials pose many new questions on risk assessment that are not yet completely answered. Thus, a reliable risk assessment related to human health and environment and safety evaluation of these materials should be performed for all in vivo studies. In 2007, the Food and Drug Administration issued a report to consider developing guidance for regulation of products that contain nanoscale materials (22).

Open Access This article is distributed under the terms of the Creative Commons Attribution Noncommercial License which permits any noncommercial use, distribution, and reproduction in any medium, provided the original author(s) and source are credited.

\section{REFERENCES}

1. Gilchrist RD, Medal R, Shorey WD, Hanselman RG, Parrott JC, Taylor CB. Selective inductive heating of lymph nodes. Ann Surg. 1957;4:596-606.

2. Marszałł MP, Moaddel R, Kole S, Gandhari M, Bernier M, Wainer IW. Ligand and protein fishing with heat shock protein 90 coated magnetic beads. Anal Chem. 2008;80:7571-5.

3. Moaddel R, Marszałł MP, Bighi F, Yang Q Duan X, Wainer IW. Automated ligand fishing using human serum albumin-coated magnetic beads. Anal Chem. 2007;79:5414-7.

4. Marszałł MP, Buciński A. A protein-coated magnetic beads as a tool for the rapid drug-protein binding study. J Pharm Biomed Anal. 2010;52:420-4.

5. Edelstein ML, Abedi MR, Wixon J. Gene therapy clinical trials worldwide to 2007—an update. J Gene Med. 2007;9:833-42.

6. Corchero JL, Villaverde A. Biomedical applications of distally controlled magnetic nanoparticles. Trends Biotechnol. 2009; 27:468-76.

7. Marszałł MP, Buciński A, Kruszewski S, Ziomkowska B. A new approach to determine camptothecin and its analogues affinity to human serum albumin. J Pharm Sci. 2010. doi:10.1002/jps. 22318.
8. Qing L-S, Xue Y, Zheng Y, Xiong J, Liao X, Ding L-S, et al. Ligand fishing from Dioscorea nipponica extract using human serum albumin functionalized magnetic nanoparticles. J Chromatogr A. 2010;1217:4663-8.

9. Alexiou Ch, Schmid RJ, Jurgons R, Kremer M, Wanner G, Bergemann Ch, et al. Targeting cancer cells: magnetic nanoparticles as drug carriers. Eur Biophys J. 2006;35:446-50.

10. Ang KL, Venkatraman S, Ramanujan RV. Magnetic PNIPA hydrogels for hyperthermia applications in cancer therapy. Mater Sci Eng C. 2007;27:347-51.

11. Dames P, Gleich B, Flemmer A, Hajek K, Seidl N, Wiekhorst F, et al. Targeted delivery of magnetic aerosol droplets to the lung. Nat Nanotechnol. 2007;2:495-9.

12. Plank Ch. Nanomagnetosols: magnetism opens up new perspectives for targeted aerosol delivery to the lung. Trends Biotechnol. 2008;26:59-63.

13. Xie Y, Zeng P, Siegel RA, Wiedmann TS, Hammer BE, Longest PW. Magnetic deposition of aerosols composed of aggregated superparamagnetic nanoparticles. Pharm Res. 2010;27:85565.

14. Alexiou C, Jurgons R, Schmid R, Hilpert A, Bergemann Ch, Parak $\mathrm{F}$, et al. In vitro and in vivo investigations of targeted chemotherapy with magnetic nanoparticles. J Magn Magn Mater. 2005;293:389-93.

15. Apopa PL, Qian Y, Shao R, Guo NL, Schwegler-Berry D, Pacurari M, et al. Part Fibre Toxicol. 2009;6:1-14.

16. Budde MD, Frank JA. Magnetic tagging of therapeutic cells for MRI. J Nucl Med. 2009;50:171-4.

17. Wilhelm C, Gazeau F. Universal cell labelling with anionic magnetic nanoparticles. Biomaterials. 2008;29:3161-74.

18. Modo M, Hoehn M, Bulte JW. Cellular MR imaging. Mol Imaging. 2005;4:143-64.

19. Bhattarai SR, Kim SY, Jang KY, Lee KC, Yi HK, Lee DY, et al. Laboratory formulated magnetic nanoparticles for enhancement of viral gene expression in suspension cell line. J Virol Methods. 2008; 147:213-8.

20. Xenariou S, Griesenbach U, Ferrari S, Dean P, Scheule RK, Cheng $\mathrm{SH}$, et al. Using magnetic forces to enhance non-viral gene transfer to airway epithelium in vivo. Gene Ther. 2006;13:154552.

21. Xiang L, Bin W, Huali J, Wei J, Jiesheng T, Feng G, et al. Bacterial magnetic particles (BMPs)-PEI as a novel and efficient non-viral gene delivery system. J Gene Med. 2007;9:679-90.

22. Nanotechnology Task Force Report. 2007, http://www.fda.gov/ ScienceResearch/SpecialTopics/Nanotechnology/Nanotechnology TaskForceReport2007/default.htm. 